\title{
Posterior lumbar interbody fusion with cortical bone trajectory screw fixation versus posterior lumbar interbody fusion using traditional pedicle screw fixation for degenerative lumbar spondylolisthesis: a comparative study
}

\author{
Hironobu Sakaura, MD, PhD, ${ }^{1}$ Toshitada Miwa, MD, PhD, ${ }^{1}$ Tomoya Yamashita, MD, ${ }^{2}$ \\ Yusuke Kuroda, MD, ${ }^{1}$ and Tetsuo Ohwada, MD1
}

'Department of Orthopaedic Surgery, Kansai Rosai Hospital, Amagasaki, Hyogo; and ²Department of Orthopaedic Surgery,
Osaka Rosai Hospital, Sakai, Osaka, Japan

OBJECTIVE Several biomechanical studies have demonstrated the favorable mechanical properties of the cortical bone trajectory (CBT) screw. However, no reports have examined surgical outcomes of posterior lumbar interbody fusion (PLIF) with CBT screw fixation for degenerative spondylolisthesis (DS) compared with those after PLIF using traditional pedicle screw (PS) fixation. The purposes of this study were thus to elucidate surgical outcomes after PLIF with CBT screw fixation for DS and to compare these results with those after PLIF using traditional PS fixation.

METHODS Ninety-five consecutive patients underwent PLIF with CBT screw fixation for DS (CBT group; mean followup 35 months). A historical control group consisted of 82 consecutive patients who underwent PLIF with traditional PS fixation (PS group; mean follow-up 40 months). Clinical status was assessed using the Japanese Orthopaedic Association (JOA) scale score. Fusion status was assessed by dynamic plain radiographs and CT. The need for additional surgery and surgery-related complications was also evaluated.

RESULTS The mean JOA score improved significantly from 13.7 points before surgery to 23.3 points at the latest follow-up in the CBT group (mean recovery rate 64.4\%), compared with 14.4 points preoperatively to 22.7 points at final follow-up in the PS group (mean recovery rate 55.8\%; $p<0.05$ ). Solid spinal fusion was achieved in 84 patients from the CBT group (88.4\%) and in 79 patients from the PS group $(96.3 \%, p>0.05)$. Symptomatic adjacent-segment disease developed in 3 patients from the CBT group (3.2\%) compared with 9 patients from the PS group $(11.0 \%, p<0.05)$.

CONCLUSIONS PLIF with CBT screw fixation for DS provided comparable improvement of clinical symptoms with PLIF using traditional PS fixation. However, the successful fusion rate tended to be lower in the CBT group than in the PS group, although the difference was not statistically significant between the 2 groups.

http://thejns.org/doi/abs/10.3171/2016.3.SPINE151525

KEY WORDS degenerative lumbar spondylolisthesis; posterior lumbar interbody fusion; cortical bone trajectory screw technique; traditional PS technique; surgical outcome; adjacent segment pathology

A $S$ a new alternative strategy to obtain improved pedicle screw (PS) fixation in the lumbar spine, a cortical bone trajectory (CBT) screw technique was advocated by Santoni et al. in 2009. ${ }^{9}$ Their cadaveric study demonstrated that the CBT screw provided a $30 \%$ increase in uniaxial yield pullout load relative to traditional PSs. ${ }^{9}$ In vivo measurement has shown that the CBT screw demonstrated 1.71-times greater insertion torque than traditional PSs. ${ }^{5}$ Several biomechanical studies have demonstrated the favorable mechanical properties of the
CBT screw. ${ }^{1,8}$ In addition, screw insertion through a caudomedial starting point enables less invasive posterior lumbar fixation by limiting dissection of the superior facet joints and reducing muscle dissection and retraction. Our cephalad screw trajectory follows a mediolateral- and caudocephalad-directed path according to the original method. ${ }^{9}$ However, our starting point of the caudal screw is on an articular surface of the superior articular process, and the trajectory takes a mediolateral path parallel to the cephalad endplate to minimize approach-related damage.

ABBREVIATIONS ASD = adjacent-segment disease; CBT = cortical bone trajectory; DS = degenerative spondylolisthesis; EBL = estimated blood loss; JOA = Japanese Orthopaedic Association; PLIF = posterior lumbar interbody fusion; PS = pedicle screw; TLIF = transforaminal lumbar interbody fusion.

SUBMITTED December 22, 2015. ACCEPTED March 23, 2016.

INCLUDE WHEN CITING Published online May 27, 2016; DOI: 10.3171/2016.3.SPINE151525. 
With respect to the surgical outcomes of posterior lumbar interbody fusion (PLIF) with CBT screw fixation, only 1 report described favorable very short-term outcomes of single-level PLIF. ${ }^{6}$ Although only 1 report examined surgical outcomes of transforaminal lumbar interbody fusion (TLIF) with CBT screw fixation compared with TLIF using traditional PS fixation, the authors showed only very short-term outcomes of TLIF in a small sample size and did not examine clinical status. ${ }^{3}$ To the best of our knowledge, no reports have examined surgical outcomes of PLIF with CBT screw fixation for degenerative spondylolisthesis (DS) compared with PLIF using traditional PS fixation in a larger patient population that had been followed-up for at least 2 years after surgery. The purposes of this study were thus to elucidate the clinical and radiological outcomes after PLIF with CBT screw fixation for DS and to compare these results with those outcomes after PLIF using traditional PS fixation.

\section{Methods \\ Patients}

Since November 2011, the lumbar spine has been fixed using the CBT screw technique in all patients who underwent PLIF at our institute because we expected that the CBT screw could have excellent fixation strength and could be inserted via a less invasive approach. Ninety-five consecutive patients who had undergone single-level PLIF with CBT screw fixation for DS since November 2011 were followed for at least 2 years after surgery (CBT group). DS was defined as more than 3-mm anterior vertebral slip on the lateral radiograph in the neutral position. All patients were considered for surgery because of unresponsiveness to conservative treatment, such as medication and/or epidural block. There were 46 men and 49 women in the study. The mean age of the patients at the time of surgery was 68.7 years (range $42-85$ years). Fusion areas were L-1 to L-2 in 1 patient, L-2 to L-3 in 1, L-3 to L-4 in 15 , L-4 to L-5 in 73, L-5 to L-6 in 1, and L-5 to S-1 in 4. The mean duration of follow-up was 35.4 months (range 25-41 months).

As a historical control group, 82 consecutive patients who had undergone single-level PLIF using traditional PS fixation for DS by the same surgeons as in the CBT group before October 2011, and were followed for at least 2 years after surgery, were enrolled (PS group). During this period, the lumbar spine was fixed using traditional PS fixation in all patients who underwent PLIF at our institute. There were 36 men and 46 women. The mean age of the patients at the time of surgery was 67.0 years (range $42-86$ years). Fusion areas were L-3 to L-4 in 7 patients, L-4 to L-5 in 72 , L-5 to L-6 in 1, and L-5 to S-1 in 2. The mean duration of follow-up was 40.2 months (range 24-61 months). There were no significant differences between the 2 groups in sex, age at the time of surgery, fusion areas, or the postoperative follow-up period (Table 1). The protocol was approved by the institutional review board of the hospital, and informed consent was obtained from all participants.

\section{Surgical Procedure}

In the CBT group, a small skin incision (about $5 \mathrm{~cm}$ ) was made at the fused segment. Dissection of facet joints
TABLE 1. Demographics of the 2 groups

\begin{tabular}{lcc}
\hline \multicolumn{1}{c}{ Variable } & CBT Group & PS Group \\
\hline No. of patients & 95 & 82 \\
\hline Male/female ratio & $46 / 49$ & $36 / 46$ \\
\hline Mean age at time of op \pm SD (yrs) & $68.7 \pm 9.5$ & $67.0 \pm 8.7$ \\
\hline Mean duration of follow-up \pm SD (mos) & $35.4 \pm 6.8$ & $40.2 \pm 10.4$ \\
\hline
\end{tabular}

and capsules supradjacent to the fused segment was limited as much as possible. On the other hand, in the PS group, paraspinal muscles and facet joints supradjacent to the fused segment were dissected to insert the traditional PS. Posterior decompression including laminotomy and partial or total facetectomy was performed in response to pathological conditions, such as combined foraminal stenosis. After intervertebral disc materials were removed, 2 carbon fiber-reinforced polyetheretherketone cages filled with local bone graft were inserted into the intervertebral space, and local bone blocks were inserted lateral to the cages. In the CBT group, our cephalad screw trajectory followed a mediolateral- and caudocephalad-directed path according to the original method. ${ }^{9}$ However, our starting point for the caudal screw was on an articular surface of the superior articular process, and the trajectory took a mediolateral path parallel to the cephalad endplate to minimize approach-related damage (Fig. 1A and B). On the other hand, the fused segment was fixed using the traditional PS procedure in the traditional PS group (Fig. 1C and D). In both groups, all PSs were inserted without an intraoperative navigation system. All patients in both groups wore a lumbosacral orthosis for 3 months after surgery.

\section{Clinical and Radiological Evaluations}

Operative duration and intraoperative estimated blood loss (EBL) were recorded as factors reflecting surgical invasiveness. Clinical results were assessed using the Japanese Orthopaedic Association (JOA) scale scoring system for assessment of the results of treatment for low-back pain (Table 2$)^{11}$ by the first author. The total JOA score is 29 points in the normal population.

Fusion status was assessed at the final follow-up by the first author. A solid fusion was defined as the condition in which osseous continuity between the vertebrae and the grafted bone was achieved on multiplanar reconstruction $\mathrm{CT}$, with neither loosening of the PSs nor motion at the fused segments on lateral flexion-extension radiographs. Fusion status was graded as either union in situ (solid fusion without loss of graft height), collapsed union (solid fusion with graft bone collapse or cage subsidence into the adjacent vertebral body), or nonunion, according to the previously reported criteria. ${ }^{10}$

For evidence of intraoperative and postoperative complications, including symptomatic adjacent-segment disease (ASD), patients' medical records were examined. Symptomatic ASD was diagnosed when clinical symptoms such as leg pain and intermittent claudication deteriorated during postoperative follow-up and the responsible lesions adjacent to the fused level were also confirmed on MRI of the lumbar spine. 



FIG. 1. Anteroposterior ( $A$ and $C$ ) and lateral view (B and $D)$ radiographs in the neutral position obtained 5 days after PLIF with CBT screw fixation ( $A$ and $B$ ) and with traditional PS fixation ( $C$ and $D)$.

\section{Statistical Analysis}

The unpaired t-test, Mann-Whitney U-test, Wilcoxon signed-rank test, Kruskal-Wallis test, and Fisher's exact probability test were used for statistical analysis with JMP (version 5.0.1, SAS Institute) as appropriate. All p values < 0.05 were considered statistically significant.

\section{Results}

\section{Clinical Results}

The mean operative duration was $123 \pm 24$ minutes (range 82-201 minutes) in the CBT group, and $145 \pm 33$ minutes (range 84-232 minutes) in the PS group, respectively ( $\mathrm{p}<0.01)$. The mean EBL was $205 \pm 152 \mathrm{ml}$ (range $10-596 \mathrm{ml}$ ) in the CBT group and $204 \pm 145 \mathrm{ml}$ (range $30-649 \mathrm{ml})$ in the PS group, respectively $(\mathrm{p}>0.05)$.

The mean JOA score improved significantly from 13.7 points before surgery to 23.3 points at the latest follow-up in the CBT group (mean recovery rate $64.4 \%$ ) compared with 14.4 points preoperatively to 22.7 points at the final follow-up in the PS group (mean recovery rate 55.8\%; Table 3). There were no significant differences in either the preoperative JOA score or the JOA score at the latest follow-up between the 2 groups. In contrast, the recovery rate of the JOA score at final follow-up was significantly higher in the CBT group than in the PS group (Table 3).

\section{Fusion Status}

In the CBT group, at the latest follow-up, union in situ was achieved in 66 of 95 patients. Collapsed union occurred in 18, and nonunion was found in 11. Solid spinal fusion was thus achieved in 84 of 95 patients (fusion rate $88.4 \%$, Table 4). In contrast, in the PS group, union in situ was achieved in 65 of 82 patients. Collapsed union occurred in 14, and nonunion was found in 3 . Solid spinal fusion was thus achieved in 79 of 82 patients (fusion rate $96.3 \%$, Table 4). The fusion rate tended to be higher in the PS group than in the CBT group, although the difference was not statistically significant $(\mathrm{p}=0.052)$.

\section{Surgery-Related Complications}

Early surgery-related complications (all complications except for ASD) occurred in 7 patients from the CBT group (7.4\%, Table 5). Regarding intraoperative complications, dural laceration occurred in 2 patients and misplacement of the PS occurred in 2 patients who needed revision surgery for replacement of the PS immediately after the initial surgery. As for early postoperative complications, 1 patient required revision surgery for evacuation of an epidural hematoma 4 days after the initial surgery. Two patients developed a superficial wound infection; both

TABLE 2. JOA scoring system for assessing the results of treatment for low-back pain

\begin{tabular}{lc}
\hline \multicolumn{1}{c}{ Item } & Point Range \\
\hline Subjective symptoms (9 points) & $0-3$ \\
\hline Low-back pain & $0-3$ \\
\hline Leg pain \&/or tingling & $0-3$ \\
\hline Gait (neurogenic intermittent claudication) & \\
\hline Clinical signs (6 points) & $0-2$ \\
\hline Straight-leg-raising test & $0-2$ \\
\hline Sensory disturbance & $0-2$ \\
\hline Motor deficit & \\
\hline Restriction of activities of daily living (14 points) & $0-2$ \\
\hline Turn over while lying & $0-2$ \\
\hline Standing & $0-2$ \\
\hline Washing & $0-2$ \\
\hline Leaning forward & $0-2$ \\
\hline Sitting (about 1 hr) & $0-2$ \\
\hline Lifting or holding heavy object & $0-2$ \\
\hline Walking & 0 to -6 \\
\hline Urinary bladder function & -6 to 29 \\
\hline Total &
\end{tabular}

* A normal total JOA score is 29 points. 
TABLE 3. Clinical outcomes in the 2 groups*

\begin{tabular}{|c|c|c|c|}
\hline \multirow[b]{2}{*}{ Group } & \multicolumn{2}{|c|}{ JOA Score (points) } & \multirow{2}{*}{$\begin{array}{c}\text { Recovery Rate of JOA score } \\
\text { at Final Follow-Up (\%) }\end{array}$} \\
\hline & Before Op & At Final Follow-Up & \\
\hline CBT & $13.7 \pm 4.6$ & $23.3 \pm 4.7 \dagger$ & $64.4 \pm 25.9 \ddagger$ \\
\hline PS & $14.4 \pm 3.9$ & $22.7 \pm 3.7 \dagger$ & $55.8 \pm 26.4$ \\
\hline
\end{tabular}

* Data given as mean \pm standard deviation.

$\dagger$ Significantly higher than before surgery (Wilcoxon signed-rank test, $p<$ 0.001).

$\ddagger$ Significantly higher than in the PS group (Mann-Whitney U-test, $p<0.05$ ).

patients were successfully treated with administration of antibiotics.

Early surgery-related complications occurred in 8 patients from the PS group (9.8\%, Table 5). As an intraoperative complication, dural laceration occurred in 3 patients and misplacement of the PS occurred in 3 patients who needed revision surgery for replacement of the PS just after the initial surgery. Regarding early postoperative complications, 1 patient required revision surgery for evacuation of an epidural hematoma 6 days after the initial surgery. One patient developed a deep wound infection; this patient was successfully treated with debridement and irrigation without removal of the implants. The incidence of early surgery-related complications showed no significant difference between the 2 groups.

Symptomatic ASD developed in only 3 patients from the CBT group (3.2\%, Table 5). Two of these 3 patients developed lumbar disc herniation and the remaining 1 patient developed foraminal stenosis at the segment adjacent to the fused area. The period between the initial surgery and the onset of symptomatic ASD ranged from 6 to 27 months (mean 17 months). All 3 patients needed additional surgery for symptomatic ASD because of unresponsiveness to conservative treatment (Table 5). In the PS group, 9 patients (11.0\%) developed symptomatic ASD (Table 5). All 9 patients developed lumbar spinal canal stenosis at the segments adjacent to the fused area. The period between the initial surgery and the onset of symptomatic ASD ranged from 10 to 46 months (mean 33 months). Eight (9.8\%) of the 9 patients needed additional surgery for symptomatic ASD because of unresponsiveness to conservative treatment (Table 5). The incidence of symptomatic ASD was significantly higher in the PS group than in the CBT group $(\mathrm{p}<0.05)$.

\section{Discussion}

The CBT screw technique is a novel lumbar PS fixation method. ${ }^{9}$ In vivo analysis by Matsukawa et al. showed that the insertion torque while placing screws using the CBT method was 1.71 times higher than that using the

TABLE 4. Fusion status in the 2 groups

\begin{tabular}{cccc}
\hline Group & Union in Situ & Collapsed Union & Nonunion \\
\hline CBT & 66 & 18 & 11 \\
\hline PS & 65 & 14 & 3 \\
\hline
\end{tabular}

TABLE 5. Surgery-related complications in the 2 groups

\begin{tabular}{ccc}
\hline Complications & CBT Group & PS Group \\
\hline Intraop (\%) & & \\
\hline Dural laceration & $2(2.1)$ & $3(3.7)$ \\
\hline Misplacement of PS & $2(2.1)$ & $3(3.7)$ \\
\hline Postop (\%) & & \\
\hline Symptomatic hematoma & $1(1.1)$ & $1(1.2)$ \\
\hline Superficial wound infection & $2(2.1)$ & $0(0.0)$ \\
\hline Deep wound infection & $0(0.0)$ & $1(1.2)$ \\
\hline Symptomatic ASD & $3(3.2)$ & $9(11.0)^{*}$ \\
\hline Additional op for ASD & $3(3.2)$ & $8(9.8)$ \\
\hline
\end{tabular}

* Significantly higher than in the CBT group (Fisher's exact probability test, $p$ $<0.05)$.

traditional PS technique. 5 Several biomechanical studies have demonstrated the favorable mechanical properties of a construct using CBT screw fixation in cadaveric lumbar specimens. ${ }^{1,8}$ Some researchers have reported that CBT screw-rod fixation provided about the same stability as traditional PS-rod fixation, regardless of the presence of interbody support. ${ }^{8}$ Others have reported that a CBT screw-rod construct has significantly superior resistance to craniocaudal toggling displacement compared with a traditional PS-rod construct. ${ }^{1}$ In addition, cranial screw insertion through a caudomedial starting point enables less invasive posterior lumbar fixation by limiting dissection of the superior facet joints and reducing paraspinal muscle dissection and retraction, which could reduce postoperative low-back pain from injury to the posteromedial branch of the nerve root crossing the superior facet joint and damage to the dissected and retracted paraspinal muscles. ${ }^{3}$ A caudomedial starting point and mediolateral- and caudocephalad-directed path of the CBT screw could also reduce the risk of superior facet violation by the screw. Regarding clinical outcomes of PLIF with CBT screw fixation, to the best of our knowledge, there has been only 1 report in the English literature that described very short-term favorable outcomes (average follow-up period 15 months) of single-level PLIF in a very small sample size $(n=12){ }^{6}$ Although only 1 report has examined surgical outcomes of TLIF with CBT screw fixation compared with TLIF using traditional PS fixation, the authors also showed only very short-term outcomes (average follow-up period in the CBT-TLIF group $=8$ months) of TLIF in a small sample size $(n=26)$ and did not examine clinical status. ${ }^{3}$ No reports have examined surgical outcomes of PLIF with CBT screw fixation for DS compared with those after PLIF using traditional PS fixation in a larger patient population that had been followed-up for at least 2 years after surgery so far. Therefore, the present study is the first to investigate the surgical outcomes after PLIF using CBT screw fixation for DS and to compare these results with those after PLIF with traditional PS fixation for DS.

With respect to clinical outcomes, the recovery rate of the JOA score at the latest follow-up visit was significantly better in the CBT group than in the PS group. However, no significant differences were shown in either the preop- 
erative JOA score or the JOA score at the latest follow-up between the 2 groups. Cranial screw insertion through a caudomedial starting point enables less invasive posterior lumbar fixation by limiting dissection of the superior facet joints and reducing muscle dissection and retraction. Moreover, our starting point of the caudal screw was on an articular surface of the superior articular process, and the trajectory took a mediolateral path parallel to the cephalad endplate to minimize approach-related damage. Although we expected that the less invasive approach associated with PLIF using CBT screw fixation could result in better clinical outcomes such as less postoperative lowback pain than PLIF using traditional PS fixation due to the reasons mentioned above, PLIF with CBT screw fixation only provided comparable postoperative recovery of clinical symptoms with PLIF using traditional PS fixation.

A higher incidence of symptomatic ASD is one of the major problems after PLIF because the incidence of symptomatic ASD is higher after PLIF than after posterolateral lumbar fusion.? In the present study, the incidence of symptomatic ASD was significantly higher in the PS group than in the CBT group. PLIF with CBT screw fixation might reduce the incidence of symptomatic ASD due to limiting the dissection of the superior facet joints, reducing muscle dissection and retraction, and possibly reducing the risk of superior facet violation, which reportedly can increase biomechanical stress and lead to destabilization at the supradjacent segment. ${ }^{2,4}$ The authors speculate that these merits of CBT screw fixation could lead to a significantly lower incidence of symptomatic ASD in the CBT group than in the PS group, although the postoperative follow-up period in the 2 groups was relatively short (the mean follow-up period was less than 5 years) and a further long-term follow-up study would thus be needed to verify a more accurate incidence of symptomatic ASD in the 2 groups.

As to fusion status, the fusion rate tended to be higher in the PS group than in the CBT group, although the difference was not statistically significant. A biomechanical study in cadaveric lumbar specimens revealed that with an intact intervertebral disc, traditional PS fixation is stiffer than CBT screw fixation during axial rotation and that with transforaminal lumbar interbody support, traditional PS fixation is stiffer than CBT screw fixation during lateral bending. ${ }^{8}$ Therefore, micromotion during axial rotation and lateral bending might result in a lower fusion rate in the CBT group than in the PS group. To improve fusion rates in the CBT group, a transverse connector might be useful by reducing micromotion during axial rotation and lateral bending.

\section{Conclusions}

Compared with PLIF using traditional PS fixation, PLIF with CBT screw fixation provided comparable postoperative improvement of clinical symptoms and could reduce the incidence of symptomatic ASD after surgery. On the other hand, the fusion rate tended to be lower after PLIF with CBT screw fixation than after PLIF using traditional PS fixation, although this difference was not statistically significant.

\section{References}

1. Baluch DA, Patel AA, Lullo B, Havey RM, Voronov LI, Nguyen NL, et al: Effect of physiological loads on cortical and traditional pedicle screw fixation. Spine (Phila Pa 1976) 39:E1297-E1302, 2014

2. Cardoso MJ, Dmitriev AE, Helgeson M, Lehman RA, Kuklo TR, Rosner MK: Does superior-segment facet violation or laminectomy destabilize the adjacent level in lumbar transpedicular fixation? An in vitro human cadaveric assessment. Spine (Phila Pa 1976) 33:2868-2873, 2008

3. Kasukawa Y, Miyakoshi N, Hongo M, Ishikawa Y, Kudo D, Shimada Y: Short-term results of transforaminal lumbar interbody fusion using pedicle screw with cortical bone trajectory compared with conventional trajectory. Asian Spine J 9:440-448, 2015

4. Kim HJ, Chun HJ, Kang KT, Moon SH, Kim HS, Park JO, et al: The biomechanical effect of pedicle screws' insertion angle and position on the superior adjacent segment in 1 segment lumbar fusion. Spine (Phila Pa 1976) 37:1637-1644, 2012

5. Matsukawa K, Yato Y, Kato T, Imabayashi H, Asazuma T, Nemoto K: In vivo analysis of insertional torque during pedicle screwing using cortical bone trajectory technique. Spine (Phila Pa 1976) 39:E240-E245, 2014

6. Mizuno M, Kuraishi K, Umeda Y, Sano T, Tsuji M, Suzuki $\mathrm{H}$ : Midline lumbar fusion with cortical bone trajectory screw. Neurol Med Chir (Tokyo) 54:716-721, 2014

7. Park P, Garton HJ, Gala VC, Hoff JT, McGillicuddy JE: Adjacent segment disease after lumbar or lumbosacral fusion: review of the literature. Spine (Phila Pa 1976) 29:19381944, 2004

8. Perez-Orribo L, Kalb S, Reyes PM, Chang SW, Crawford NR: Biomechanics of lumbar cortical screw-rod fixation versus pedicle screw-rod fixation with and without interbody support. Spine (Phila Pa 1976) 38:635-641, 2013

9. Santoni BG, Hynes RA, McGilvray KC, Rodriguez-Canessa G, Lyons AS, Henson MA, et al: Cortical bone trajectory for lumbar pedicle screws. Spine J 9:366-373, 2009

10. Yamamoto T, Ohkohchi T, Ohwada T, Kotoku H, Harada N: Clinical and radiological results of PLIF for degenerative spondylolisthesis. J Musculoskelet Res 2:181-195, 1998

11. Yone K, Sakou T, Kawauchi Y, Yamaguchi M, Yanase M: Indication of fusion for lumbar spinal stenosis in elderly patients and its significance. Spine (Phila Pa 1976) 21:242248, 1996

\section{Disclosures}

The authors report no conflict of interest concerning the materials or methods used in this study or the findings specified in this paper.

\section{Author Contributions}

Conception and design: all authors. Acquisition of data: Sakaura. Analysis and interpretation of data: Sakaura. Drafting the article: Sakaura. Critically revising the article: Sakaura, Miwa, Yamashita, Kuroda. Reviewed submitted version of manuscript: Sakaura, Miwa, Yamashita, Kuroda. Approved the final version of the manuscript on behalf of all authors: Sakaura. Statistical analysis: Sakaura. Study supervision: Ohwada.

\section{Correspondence}

Hironobu Sakaura, Department of Orthopaedic Surgery, Kansai Rosai Hospital, 3-1-69 Inabaso, Amagasaki, Hyogo 660-8511, Japan.email: sakaura04061023@yahoo.co.jp. 